Patient Education and Counseling, 7 (1985) 157-165

Elsevier Scientific Publishers Ireland Ltd.

\title{
EVALUATION OF A MEDICATION REFILL REMINDER SYSTEM FOR A COMMUNITY PHARMACY
}

\author{
FRANK J. ASCIONE ${ }^{\mathrm{a}}$, GRANT H. BROWN ${ }^{\mathrm{b}}$ and DUANE M. KIRKING ${ }^{\mathrm{c}}$ \\ ${ }^{a}$ College of Pharmacy, University of Michigan, ${ }^{b}$ Brown's Pharmacy, Jackson and College \\ of Pharmacy and School of Public Health, University of Michigan, Ann Arbor, MI 48109- \\ 1065 (U.S.A.)
}

(Received July 30th, 1984)

(Accepted November 8th, 1984)

\section{ABSTRACT}

The problem of maintaining compliance in patients taking chronic medications is well established. One recommended approach to reducing this problem is to develop a system which identifies non-compliers in order that interventions can be devised to improve that behavior. A medication refill reminder system is an example of this approach.

The refill behavior was observed for 102 cardiovascular patients who were patrons of one community pharmacy in order to assess the effects of a postcard/telephone reminder system. Patients who failed to refill their prescriptions on time were sent a postcard reminding them to have them refilled. A telephone call would follow if the patient failed to visit the pharmacy after the postcard.

The study results showed that the average days late for patients who failed to refill their prescription as expected decreased from 19.15 to 6.6 days after the reminder system was used $(P<0.005)$. In addition, the percent of late refills of these patients was $13 \%$ less than controls. These findings provide strong evidence that a refill reminder system can be used successfully in a community pharmacy as part of a program to identify non-compliant patients and improve their compliance with chronic medication therapy.

Key words: Refill reminder system - Patient medication compliance Telephone/postcard reminders

\section{INTRODUCTION}

Patients taking medications for long periods of time are likely to experience difficulty in taking them correctly throughout that period. The rate of 0738-3991/85/\$03.30 $\odot 1985$ Elsevier Scientific Publishers Ireland Ltd. 
compliance tends to decrease in these patients as the length of therapy increases [1]. Health professionals have a responsibility to try to prevent the expected decline in compliance rates in patients taking medications on a chronic basis. It has been suggested that close and continued supervision of patient compliance may be an effective general strategy to modify that behavior [2-4].

One specific system that may be an effective means of monitoring parient long-term compliance behavior is a postcard/telephone reminder system. This approach has been used successfully in numerous studies of appointment keeping [5]. Similar success has occurred in studies of medication-refill behavior. An automatic telephone callback system improved the on-time refill behavior of cardiovascular patients receiving digitalis preparations by $10 \%$ during a 1-year period [6]. A reminder postcard sent to tuberculosis patients who were late in refilling their isoniazid prescription resulted in $53 \%$ of the patients responding to the refill reminder [7]. A similar positive response (59\%) to a reminder postcard occurred in patients undergoing therapy for a number of chronic health problems (e.g. hypertension, congestive heart failure, diabetes) [8]

The various studies described used outpatients seeking care at a clinic or hospital. There is little research available on the utility of a medication refill reminder system in a community pharmacy. Because approx. $70 \%$ of all patients receive their prescription medications from one community pharmacy [9], and tend to visit that pharmacy more often than they are seen by their physician [10], it is a logical place to monitor patient-refill behavior. A brief report [11] of a computerized refill reminder system used in three community pharmacies indicated that such a system may be effective. In this study, patients in the experimental group automatically received a reminder telephone call 3 days prior to their calculated refill date. This approach resulted in medications being obtained in significantly fewer days late for the experimental group compared to controls.

While there is adequate documentation that a medication refill reminder system is likely to improve the refill behavior of patients receiving chronic medication, some questions about this system are not yet answered. None of the studies used a combination of postcard and telephone calls as a means of encouraging patient-refill behavior. In addition, the studies usually focused on the refill behavior for short periods of time, usually a month. Finally, despite the success of the computerized telephone reminder system used in the three community pharmacies [11], there is little known about the value of other types of medication-refill reminder systems in these facilities.

This study attempted to address those issues by examining the utility of a postcard/telephone refill reminder system in a community pharmacy. The specific study objectives were to (1) assess the effectiveness of a reminder system in improving the refill behavior of patients who fail to refill their prescriptions on time and (2) determine the feasibility of utilizing a medication reminder system in a busy community pharmacy. 


\section{METHODS}

The study setting was a community pharmacy in a southeastern Michigan city of approx.40,000 people. The pharmacy provided services to a primarily white middle class patient population and filled 75-100 prescriptions per day while being open $48 \mathrm{~h} /$ week. Third-party billing services were provided for all programs and accounted for approx. $40 \%$ of all prescriptions.

Patients were asked to participate in the study when they came into the pharmacy for a new or refill prescription for a cardiovascular medication (CVM). Nine categories of CVMs were included such as antiarrhythmics, antihypertensives and antiangina drugs. Patients were eligible if they were at least 30 years of age and taking a minimum of one CVM on a regularly scheduled basis. In addition, they had to be stabilized on the drug for at least 3 months. Patients were not to be absent from the area for a period of greater than 34 days and were to be able to assume responsibility for taking their own medication.

Cardiovascular medications were selected because they represent a large group of medications that are taken by a diverse patient population and for which the importance of patient compliance with the dosing regimen is well recognized. Patients were excluded if they were receiving a CVM from more than one pharmacy or refused to sign the consent needed to participate in the study.

After their consent was obtained, patients were randomly allocated into two groups. The first group consisted of individuals who acted as controls and whose refill behavior was only observed during the study period. The second group consisted of individuals who would receive a reminder if they were late refilling their prescriptions. All patient refill behavior was followed for 4 months.

The patients participated in an extensive initial interview about their medications with a pharmacist. The pharmacist asked the patient to recall the name of each medication they were taking, describe its purpose, how it was to be taken and if they could name common side effects associated with the drug. Factors that may result in missing a dose of their medication, action to be taken if a dose was missed and storage of their medications were also discussed. The interview could be done at the time the prescription was filled or the patients could return to the pharmacy within 1 week and complete the interview at a time appointed by the pharmacist. This standardized counseling procedure assessed each patient's drug knowledge, verified information from the patient's medication profile, reinforced important points concerning the medication regimen, and minimized the variability during the pharmacist-patient exchange.

Follow-up discussions were conducted as necessary each time the patient returned to the pharmacy. Topics covered in these discussions could be the result of questions by the patient or reinforcement of areas discussed during the initial review.

The medication reminder system consisted of an index file with cards for 
each day of the year, a calendar of consecutively numbered days of year, and a drug profile card. When a patient in the experimental group entered the reminder system, a drug card was placed into the index file at the date corresponding to 2 days prior to the calculated refill date. This was done for each CVM the patient was taking, thus allowing the pharmacist to anticipate when the patient was due to return to the pharmacy for a refill of a given prescription.

When the patient returned to the pharmacy on time, the prescription was refilled and the drug profile card was forwarded in the index file to the next calculated refill date. If the patient did not return to the pharmacy within 3 days after the calculated refill date a reminder card was mailed to the patient. The patient was contacted by telephone if the prescription had not been refilled within 10 days of the calculated refill date.

\section{Development of interventions}

The interventions used during this study were the reminder postcard, telephone and follow-up discussions with the patient. These interventions were monitored and conducted by a trained pharmacy technician and the three pharmacists working in the pharmacy.

The purpose of the medication reminder system was to identify individuals with unexpected refill behaviors and contact those individuals to determine why this behavior was inconsistent with the refill record. If the failure to refill a prescription was due to a change in dosage by the physician or an extra supply of medication, then the prescription record could be corrected to reflect the actual, appropriate refill behavior. Conversely, if the unexpected refill behavior was due to actual non-compliance, the postcards and telephone call would serve as a reminder or cue to the patient to comply.

The focus of this reminder system on problem identification is different than the focus of the system used in the other community pharmacy study [11]. The previous system was designed to remind patients of the need for refills prior to the expected refill date. Thus, all patients would receive a reminder even if they actually planned to refill the prescription on time. While this approach may be successful in improving patient-refill behavior, it is time consuming and requires advance information with regards to when each patient is expected to refill their prescriptions. In addition, the constant exposure to reminders may be viewed negatively by the patient. However, the use of the reminder system as a method to identify those patients who actually plan to not refill their prescription on time is much more efficient and less likely to receive a widespread negative patient reaction.

When follow-up discussions were conducted with patients, the information discussed was recorded on pre-printed sheets. The discussion was facilitated by the use of patient-medication profiles for both groups and also the medication reminder system for the experimental group. The purpose of these discussions was to elicit patient reaction to the reminder system.

\section{Measures}

Refill behavior was monitored by following the refill frequency of a 
medication with the use of the patient profile and specific information regarding each prescription. This information included the date the prescription was filled, the quantity given at the time of refill, the days supply for that prescription if taken as prescribed, and the refill date of the prescription. From this information, the pharmacist could determine if the prescription was refilled early, on time, or late. Prescriptions refilled within 3 days of the calculated refill date were considered to be on time, while those refilled more than 3 days before or after the calculated refill date were considered early or late, respectively. By determining the status of the prescription refill with the patient profile for the control group and the medication-reminder system for the experimental group, the pharmacist could evaluate if the patient was exhibiting appropriate refill behavior for the medication. This procedure also allowed the investigators to evaluate the influence of the reminder system on this behavior.

Once possible late refill behavior was identified, the pharmacist discussed with the patient the reason(s) for the late refills. These discussions were completed when the patients returned to the pharmacy or by telephone if necessary. The results of these discussions were recorded on the follow-up sheet.

After patients participated in the study for 4 months, a questionnaire was mailed to them assessing their attitudes and responses to the services provided. It served to introduce the reminder system to patients in the control group and those in the experimental group who had not received a reminder postcard.

\section{RESULTS}

A total of 120 patients were invited to participate in the study. Six patients $(5 \%)$ refused and 12 patients $(10 \%)$ completed the questionnaire but did not return to the pharmacy for the initial interview or were found to not meet the study screening criteria. This resulted in 102 patients $(85 \%)$ between 31 and 98 years of age participating in the study. The average age of the study group was 63 years and was $61 \%$ female. The patients were taking an average of 4.8 drugs (2.3 of which were CVMs).

Overall, vasodilators were the most frequently prescribed class of medication (19.6\% of the medications taken) and propranolol was the most frequently used individual medication (10.6\% of the medications taken). The range of medications taken in the study population was $1-8 \mathrm{CVMs}$ and from 0 to 11 non-cardiovascular medications. The most medications (both cardiovascular and non-cardiovascular) taken was 16.

Twenty-eight patients (27.0\%) completed the initial interview at the time their prescription was being filled, while 74 patients $(73.0 \%)$ returned at a later time to the pharmacy to participate in the interview process. The interviews averaged $23 \mathrm{~min}$ in length with a range of 7-60 min.

Table I indicates that a significant number of the patients taking cardiovascular medications were late in having their prescriptions refilled during the 4 -month period that they were being followed. Sixty-three $(62 \%)$ of the 
TABLE I

PATIENT REFILL BEHAVIOR

\begin{tabular}{lll}
\hline Measures & No. & Percent $^{\mathbf{a}}$ \\
\hline No. of patients with late refills & & \\
$(N=102)$ & & 26.5 \\
$\quad$ Control & 27 & 35.3 \\
Experimental & 36 & 61.8 \\
$\quad$ Combined & 63 & \\
Average days late/refill per drug & \\
$\quad$ Control $(N=258)$ & & \\
Experimental $(N=242)$ & 4.31 & \\
Combined $(N=500)$ & 2.63 & 3.41 \\
\hline
\end{tabular}

apercent of total patients in the study.

${ }^{b}$ Late refills were those refills greater than 3 days past the expected refill date.

${ }^{c} N=$ total number of drugs taken by patients in that group.

102 patients failed to refill at least one of their prescriptions on time. The patients filled $26 \%$ of their prescriptions late, averaging 5.49 days beyond the expected refill date. Of the 237 drugs these patients were taking, 88 (37\%) were refilled late. In addition, $30(57 \%)$ of the 52 patients in the experimental group received a reminder postcard and 13 patients (25\%) also required a follow-up telephone call. Because six patients who were greater than 5 days late for a refill did not receive a reminder postcard due to clerical error, the actual number of patients who should have received a postcard was $36(69 \%)$ of the experimental group.

The results as shown in Table II indicate that the reminder system was effective in improving patient-refill behavior. Those patients in the experimental group who had more than one late refill and received a reminder card experienced a highly significant decrease (nearly 300\%) in the days late between their first and second refills $(P<0.005)$. The refill behavior remained improved throughout the study period. In addition, the percentage of late refills for 'late' patients (i.e. those who had one or more late refills) was

TABLE II

EFFECT OF REMINDER POSTCARD ON REFILL BEHAVIOR

\begin{tabular}{lccc}
\hline Refill No. & $N$ & Average days late/refill & Significance $^{\mathrm{a}}$ \\
\hline $1^{\mathrm{b}}$ & 20 & 19.15 & - \\
2 & 20 & 6.60 & $P<0.005$ \\
3 & 16 & 7.25 & $P<0.05$ \\
4 & 9 & 3.56 & $P=0.09$ \\
\hline
\end{tabular}

${ }^{a}$ Statistical comparisons are between refill 1 and refills 2,3 and 4, respectively. Comparisons were made with a paired $t$-test.

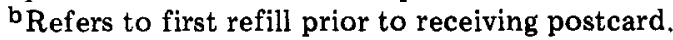


evaluated by a chi-squared test. It was determined that the control group had late refills $53 \%$ of the time while the experimental group had late refills $41 \%$ of the time. The $13 \%$ difference between the two groups was not statistically significant due to the small sample size $(N=19)$. However, the trend indicated an influence on patients receiving reminder postcards.

The patients provided 56 reasons for not refilling their prescriptions on time. Twenty-one reasons (38\%) involved having an extra supply on hand from various sources such as physicians' samples, sharing the medications with others, or the dose being decreased by the doctor. The medication was discontinued by the patient's physician in nine cases $(16 \%)$. In only 6 instances $(10 \%)$ was the incorrect refill behavior reported to be due to intentional or unintentional non-compliance with the medication regimen. Miscellaneous reasons such as: could not afford medications; card being sent at the wrong time; refill record information incorrect were given in 20 cases $(37 \%)$.

\section{DISCUSSION}

In the patient population followed during the study, a need for careful monitoring of refills was indicated by the large portion of the patients $(62 \%)$ displaying late refill behavior. This result is similar to findings of three other studies which reported that between $51-60 \%$ of the patients studied were late in refilling their prescriptions [6-8]. Like those other studies, a refillreminder system was effective in modifying that behavior.

It was also shown that exposure to the combination of reminder postcards and a telephone call may be useful in modifying the refill behavior of patients unresponsive to a single reminder method. The rate of patients unresponsive for a single reminder in other studies was quite large, ranging from 17 to $47 \%$ [6-8]. In this study, 7 of the 20 persons (35\%) with subsequent refills needed to be telephoned because they failed to respond to the original postcard. Five of those patients refilled their prescriptions within the 5-day limit on subsequent refills.

A medication-reminder system such as the one utilized in this study should also be considered an aid to the pharmacist in identifying and reducing medication-related problems in patients taking chronic medication. Although a majority of the incorrect refill situations did not represent noncompliance as admitted by the patient, several of these problems, such as sharing of medication with others, represented situations where a corrective intervention was indicated. While some of the patients may have been purposely or unconsciously misleading about the physician changing the dose regimen, a major value of the reminder system for these individuals is the correction of the pharmacy record to more accurately reflect how the patient is taking the drug. This updated information should then be confirmed with the physician's record. Once these adjustments are recorded, the pharmacist should be better able to monitor the patient's future refill behavior. 
While not as frequent, there were a number of patients identified who admitted taking their medications inappropriately. The fact that this behavior is infrequent would make detection difficult without a systematic problemidentification system such as the one used in this study. Thus, a major benefit of this system may be in assisting the pharmacist and other health professionals in identifying relatively uncommon chronic-care patients who do not comply.

Patients' acceptance of the use of the reminder system was both positive and negative. Better pharmacist awareness of the patient's refill frequency and the improved pharmacist ability to identify potential problems were included in the positive responses. Negative responses included the feeling that patients should be responsible for their own interests and did not need to be reminded of that responsibility by the pharmacist.

This system, while not only effective, is also feasible to implement in a community pharmacy practice. The initial development of the program and the entering of patients into the system was the most time-consuming of the activity. Once the reminder system was operational, it required approx. 14 min daily to maintain the system for 50 patients, $70 \%$ of which was clerical time. Operation of the present system was handled by existing personnel at an estimated time cost of $\$ 1.75 /$ day. Daily supplies (e.g. postcards, postage) and telephone costs were about 15 cents. Thus, the whole system could be maintained for a cost of about 4 cents/patient per day. Obviously, the total amount of time and costs spent operating the system would be much greater if a particular pharmacy monitored all its chronic-care patients in this manner. It would be prudent, therefore, to target specific groups of patients who are likely to need the service most and implement the service on a priority basis. For example, the target group in this study was those patients who were taking cardiovascular medications. These individuals were selected because of the potency of the medications they were taking and the increased risk of medication-related problems if they fail to take the drug properly.

Potential benefits of the system to the pharmacist other than problem identification and resolution do exist although they were not examined in this study. They include the ability to refill prescriptions for chronic medications in advance of the patient's return. This activity takes advantage of less busy times in the pharmacy and thereby decreases patient waiting time. Increased patronage may result from better compliance and increased patient satisfaction [11]. In addition, the potential for a significant minority of patients to pay for this type of service should not be ignored [13].

Finally, computerization of the reminder system could decrease operating costs significantly by automatically identifying overdue refills and generating reminder postcards. Such a program could operate from the data which is currently entered into existing computer systems. It is estimated that over $25 \%$ of community pharmacies are computerized today [12] and that all should be using this technology in the future. Thus, a reminder program may be a useful feature to be included in these systems. 


\section{ACKNOWLEDGMENTS}

Work was carried out at the University of Michigan College of Pharmacy. The authors acknowledge Isabelle Brown, RPh, Denniston Brown PPh, and the staff of Brown's Pharmacy, Jackson, Michigan for their assistance in this study.

\section{REFERENCES}

1 Haynes RB. introduction. in Haynes RB, Taylor DW, Sackett DP (Eds.), Compliance in Health Care. Johns Hopkins University Press, Baltimore, 1979, 18.

2 Haynes RB, Sackett DL. Gibson ES, Taylor DW et al. Improvement of medication compliance in uncontrolled hypertension. Lancet $1976 ; 1: 1265$.

3 McKenney JM, Brown ED, Necessary R, Reavis HL. Effect of pharmacist drug monitoring and patient education on hypertension patients. Contemp Pharm Pract 1978; $1: 50$.

4 McKenney JM, Slining JM, Henderson HR, Devins D et al. The effect of clinical pharmacy services on patients with essential hypertension. Circulation 1973; $48: 1104$.

5 Haynes RB. Strategies to improve compliance with referrals, appointments, and prescribed medical regimens, in Haynes RB, Taylor DW, Sackett DL (Eds.), Compliance in Health Care, Johns Hopkins University Press, Baltimore, 1979, pp. 123126.

6 Canada AT. The pharmacist and drug compliance. in Sackett DL and Haynes RB (Eds.), Compliance with Therapeutic Regimen. Johns Hopkins University Press, Balitimore, 1976, pp. 131-134.

7 Ellinoy BJ, Mays JF, McSherry PV, Rosenthal LC. A pharmacy outpatient monitoring program providing primary medical care to selected patients. Am J Hosp Pharm 1973; $30: 593$.

8 Brown DJ, Ellsworth A, Taylor JW. Identification of potentially noncompliant patients with a mailed medication refill reminder system. Contemp Pharm Pract $1980 ; 3: 244$

9 Upjohn Co. National Pharmacy Service Study. Market Facts Inc., Chicago, 1979.

10 Shimp LA, Ascione FJ, Glazer HM. A Community Based Program to Reduce Medication-related Problems in Non-institutionalized Adults. Michigan Office of Service to the Aging Report, 1984.

11 Bryan RA, Jackson, RA, McKay AB. A Cost-Benefit Analysis of Computer Identified Telephone Refill Reminders in Community Pharmacy. Presented before the American Pharmaceutical Association Academy of Pharmacy Practice, April 1983.

12 Lockwood WA, Bauman NR. ComputerTalk Directory of Pharmacy Systems. ComputerTalk Associates, Blue Bell, PA, 1983, p. 4.

13 Brown GH, Kirking DM, Ascione FJ. Patient willingness to pay for a community pharmacy based medication reminder system. Am Pharm 1983; NS23 :69. 